\title{
Van Velthoven single-knot running suture versus Chlosta's running suture versus single barbed suture V-Loc for vesicourethral anastomosis in laparoscopic radical prostatectomy: a retrospective comparative study
}

\author{
Tomasz Wiatr ${ }^{1}$, Lukasz Belch ${ }^{1}$, Katarzyna Gronostaj ${ }^{1}$, Dominik Choragwicki ${ }^{1}$, Anna K. Czech ${ }^{1}$, Lukasz Curylo ${ }^{1}$, \\ Jakub Fronczek², Mikolaj Przydacz ${ }^{1}$, Przemyslaw Dudek ${ }^{1}$, Piotr Chlosta ${ }^{1}$ \\ ${ }^{1}$ Department of Urology, Jagiellonian University Medical College, Krakow, Poland \\ ${ }^{2}$ Department of Intensive Care and Perioperative Medicine, Jagiellonian University Medical College, Krakow, Poland
}

Videosurgery Miniinv 2022; 17 (1): 214-225 DOI: https://doi.org/10.5114/wiitm.2021.105851

\begin{abstract}
Introduction: The quality of vesicourethral anastomosis (VUA) in laparoscopic radical prostatectomy (LRP) is associated with complications that could significantly affect quality of life.

Aim: To compare different types of sutures (Chlosta's versus Van Velthoven versus V-Loc), used for VUA in LRP in terms of complication rates and continence recovery.

Material and methods: Patients who underwent LRP between 2014 and 2018 in a tertiary center were enrolled in the study. Data were extracted from medical records. Urinary continence was assessed at 3, 6, 12 and 18 months after LRP. Propensity score weighted regression models were used to estimate the effect of sutures on outcomes.

Results: A sample of 504 patients was analyzed, of which 109 patients underwent Chlosta's suture VUA, 117 patients had Van Velthoven suture VUA, and 278 patients had V-Loc VUA. Median time of anastomosis was 13 (IQR - interquartile range: 10-16) min using Chlosta's suture, 28 (IQR: 24-30) using Van-Velthoven suture and 12 (IQR: 11-16) min using $V$-Loc suture $(p<0.001)$. There were no significant differences between groups concerning complications and urinary continence at 12 and 18 months after surgery. The time of urinary continence recovery was on average 19 days (95\% Cl: 5-33) and 31 days (95\% Cl: 16-45) shorter during 1 year of observation when the V-LoC suture was used compared to the Van-Velthoven and Chlosta's suture, respectively.

Conclusions: The study showed comparable results considering urinary continence recovery at 12 and 18 months after LRP in all VUA groups. Van Velthoven VUA was more time-consuming and continence recovery was faster in the $V$-Loc group.
\end{abstract}

Key words: prostate cancer, laparoscopic radical prostatectomy, vesicourethral anastomosis.

\section{Introduction}

Prostate cancer (PCa) is one of the most commonly diagnosed cancers in men. In the United States and many Western European countries, the incidence of PCa outweighs the incidence of other malignancies among the male population [1]. PCa remains the second leading cause of death in males. Since 1985, a gradual increase in PCa-related mortality has been observed worldwide [2]. 
Laparoscopic radical prostatectomy (LRP) was first introduced by Schuessler et al. in 1997 [3]. It is a minimally invasive surgical technique that is offered to men with localized PCa. Endoscopic treatment of PCa offers good visibility of the surgical field, which leads to more accurate identification of anatomical structures [4-6].

Despite the technical improvement, it should be remembered that LRP is associated with the risk of intra- and postoperative complications and requires surgical skills in laparoscopic techniques. This method represents a challenge for many urologists, especially considering the learning curve, procedure difficulty, and time needed to perform vesicourethral anastomosis (VUA) [7]. The quality of VUA is associated with complications that could significantly affect the patient's urinary-related quality of life, such as urethral stricture, bladder neck contracture (BNC), or urinary incontinence. The most popular and widely used VUA technique is the method introduced in 2003 by Van Velthoven et al. [8]. Despite its effectiveness, this method is time-consuming (average anastomosis time is $35 \mathrm{~min}$ ) and not free of complications. Many VUA methods during LRP aim to simplify the surgical procedure and reduce the time of surgery [9-16]. In 2009, an alternative technique for laparoscopic running VUA, Chlosta's running suture, was described $[17,18]$. The aim of the current study was to compare complication rates and urinary function outcomes of patients who underwent LRP in terms of the VUA suturing technique in a single tertiary care center in Poland.

\section{Aim}

To compare three different types of sutures (Chlosta's running suture, Van Velthoven single-knot running suture, V-Loc suture) that are used for VUA in LRP in terms of complication rates and urinary continence recovery.

\section{Material and methods}

Patients who underwent LRP between February 2014 and October 2018 in a single tertiary care center in Poland were enrolled in this retrospective study. The exclusion criteria were as follows: patients with a history of radiotherapy for PCa or distant metastases; preoperative stress-, urge, or mixed urinary incontinence; or a history of urethral trauma or stricture. Also excluded were patients with previ- ous pelvic surgery, which might have affected their voiding function.

The study was approved by the local ethics committee. All operations were performed by four experienced surgeons, all of whom had performed more than 100 LRPs prior to the study. To evaluate the potential issue of operator bias in our study, we performed interaction tests between the operating surgeon and the effect of sutures on suturing time and the occurrence of complications.

Preoperative and perioperative data were extracted from medical records. Patients' urinary continence status after catheter removal was assessed at 3, 6, 12, and 18 months after LRP. Urinary continence recovery was defined as the use of zero pads. The incidence of BNC was assessed concomitantly with urinary continence status up to 18 months after LRP. Complications were assessed within a 90day postoperative period in accordance with the Clavien-Dindo classification [19].

\section{Surgical techniques}

\section{Group 1: Chlosta's running suture}

After the urethra was cut off and full hemostasis achieved, VUA was performed with a single running suture using the center's own modification. This

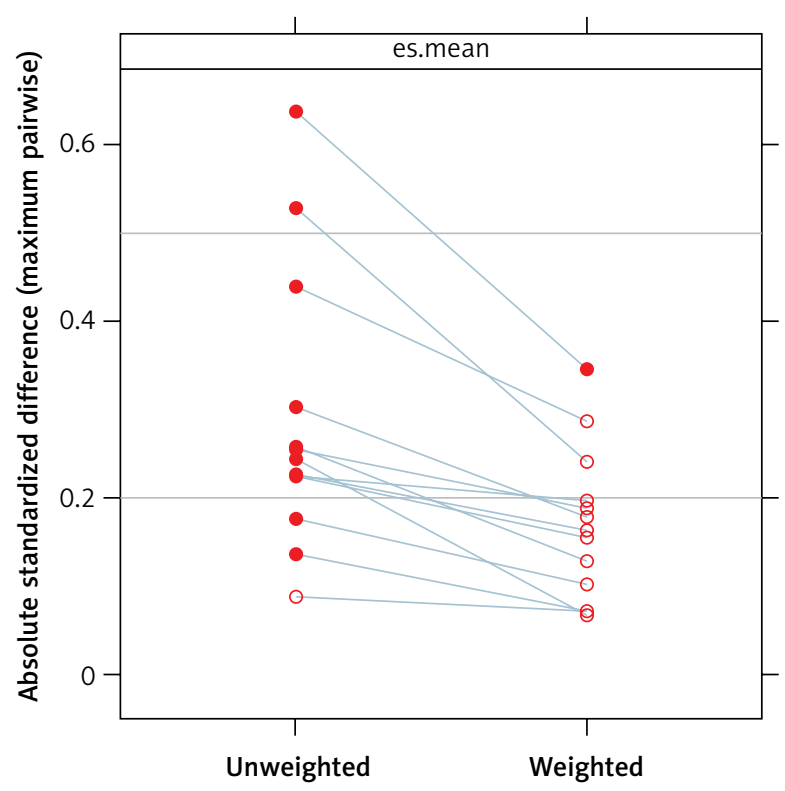

Figure 1. Standardized effect size of variables included in the propensity score model. Effect of weights on the magnitude of differences between treatment group 

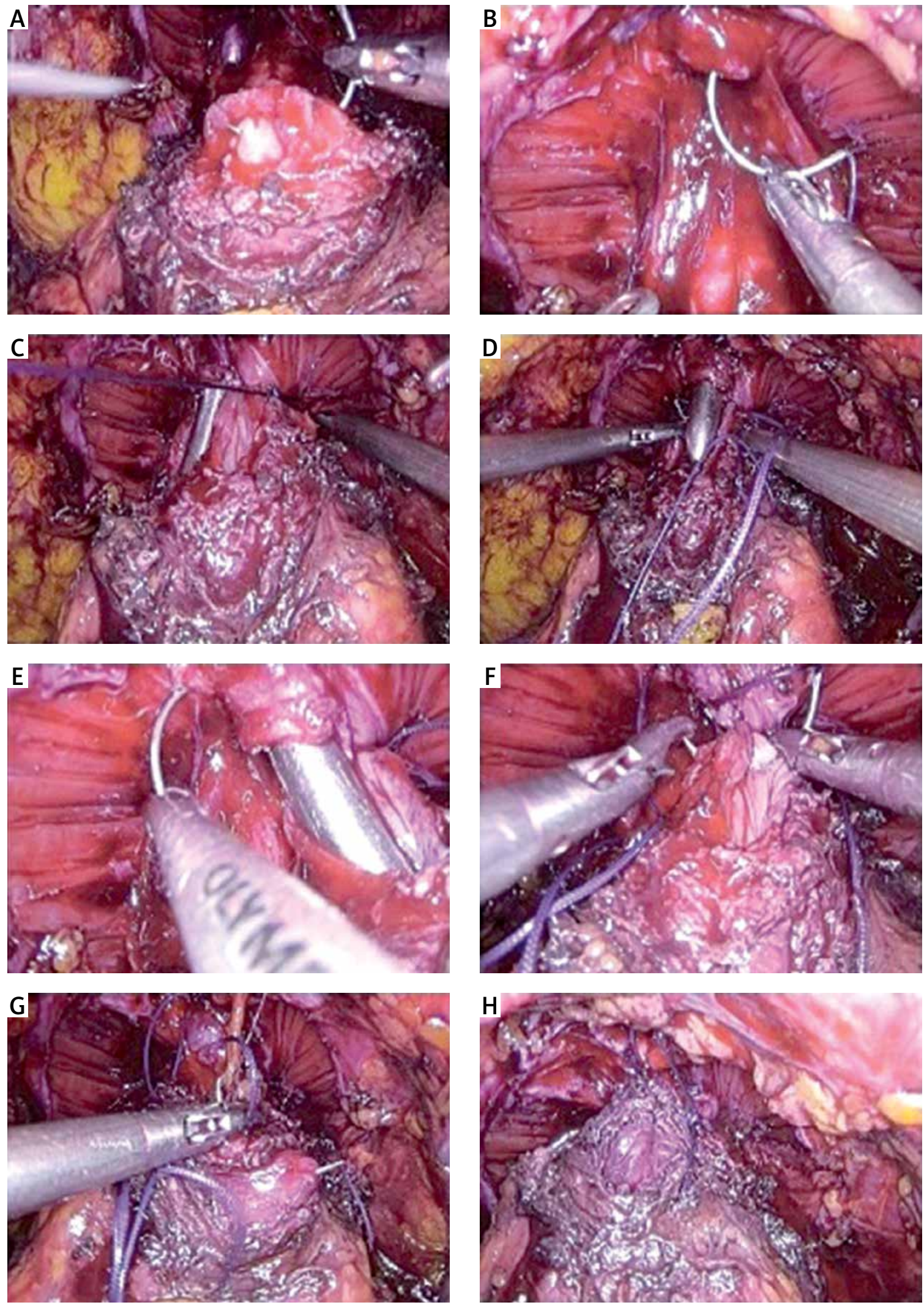

Photo 1. Technique for performing Chlosta's running suture 
method involved the use of a continuous suture (polyglactin 2-0, absorbable synthetic suture), which was placed first at the 5 o'clock position on the bladder neck outside-in and then inside-out toward the urethra, and the suture was tied. The continuous suture was then continued counterclockwise on the bladder neck outside-in and inside-out on the urethra and a tight anastomosis performed so that it covered the full thickness of the bladder wall and urethra. On average, five to seven needle passes were placed on both the bladder neck and the urethra until the free end of the suture at 5 o'clock was met. Just before the final suture of the anastomosis (Figure 1, Photo $1 \mathrm{G}$ ), an $18 \mathrm{~F}$ silicone Foley catheter was introduced into the bladder under the surgeon's direct vision. After both ends of the suture were tied, the watertightness of the anastomosis was assessed by filling the bladder with $100-200 \mathrm{~cm}^{3}$ of sterile physiological saline. If necessary, an additional suture was placed to avoid urinary leakage. After completion of the anastomosis, the balloon of the catheter was filled with $10 \mathrm{ml}$ of sterile water. A 20F Redon drain was placed. In all patients with drainage output over $100 \mathrm{~cm}^{3}$ during the first $8 \mathrm{~h}$ after surgery, the creatine level of the fluid was assessed. The decision on catheter removal was at the discretion of the surgeon.

\section{Group 2: Van Velthoven suture}

After laparoscopic removal of the prostate, the bladder neck was identified. The running suture was prepared extracorporeally by tying together the ends of two 2-0 polyglycolic sutures, one dyed and one not, for identification purposes. The running suture was initiated by placing both needles outside-in through the bladder neck and inside-out on the urethra, one needle at the 5:30-o'clock position and the other at the 6:30-o'clock position. After three additional needle passes (bladder neck-urethra-bladder neck) symmetrically, counterclockwise with one thread and clockwise with the other, gentle traction was applied on each thread. At this point, an $18 \mathrm{~F}$ silicone catheter was placed in the bladder. The suture was then continued until both threads reached the $12 o^{\prime}$ 'clock position, outside-in on the bladder neck and inside-out on the urethra. To avoid tying the knot on the urethral side, the surgeon placed the last needle pass on either thread outside-in on the urethra and then inside-out on the bladder neck, after which the suture was tied. If maladjustment persisted between the diameters of the urethra and the bladder neck, the remaining anterior opening of the bladder neck was closed with 2-0 polyglycolic sutures (Photo 2). After the VUA was completed, the watertightness of anastomosis was assessed by filling the bladder with $100-200 \mathrm{~cm}^{3}$ of sterile physiological saline. The balloon of the catheter was filled with $10 \mathrm{ml}$ of sterile water. A 20F Redon drain was placed. In all patients with drainage output over $100 \mathrm{~cm}^{3}$ during the first $8 \mathrm{~h}$ after surgery, the creatinine level of the fluid was assessed. The decision on catheter removal was at the discretion of the surgeon.

\section{Group 3: Single barbed suture (V-Loc)}

During VUA, a loop at the end of the V-Loc suture was used to anchor it to the neck of the bladder without the need for a knot. Moreover, while using this suture, the tissues of the bladder neck and urethra were opposed and secured in position after each needle passage, reducing the need for repeated suture tightening and tissue traction. The VUA was started on the bladder neck in the 5 o'clock position outside-in, and then the needle was passed through the urethra inside-out. A second needle pass was placed back on the bladder neck at the 6 o'clock position and from there it ran counterclockwise through the urethra and bladder neck. After a third pass with the needle through the bladder neck, gentle thread traction was applied to bring the bladder and urethra together. At this stage, an $18 \mathrm{~F}$ Foley catheter was placed in the bladder. The entire anastomosis was performed with five to seven needle passes on both the urethra and the bladder neck. After finishing the VUA, the surgeon assessed the watertightness of anastomosis by filling the bladder with $100-200 \mathrm{~cm}^{3}$ of sterile physiological saline (Photo 3). The balloon of the catheter was filled with $10 \mathrm{ml}$ of sterile water. A 20F Redon drain was placed. In all patients with drainage output over $100 \mathrm{~cm}^{3}$ during the first $8 \mathrm{~h}$ after surgery, the creatinine level of the fluid was assessed. The decision on catheter removal was at the discretion of the surgeon.

\section{Statistical analysis}

No sample size calculations were performed before initiating the study. Descriptive statistics on baseline variables are presented as median (interquartile range (IQR)) or count and percentage. Between-group differences were investigated using 

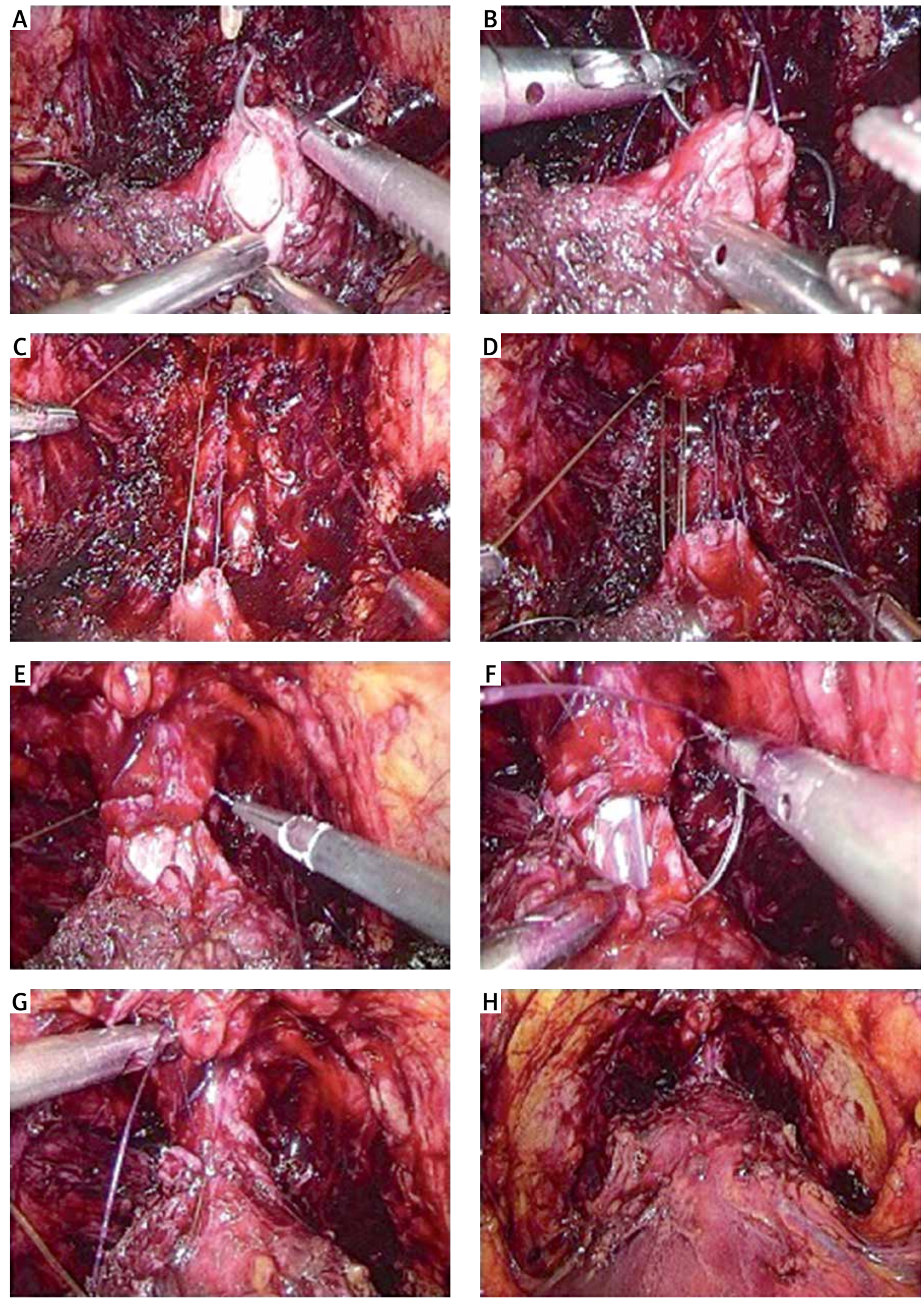

Photo 2. Anatomical restoration of the vesicourethral junction with the Van Velthoven suture 

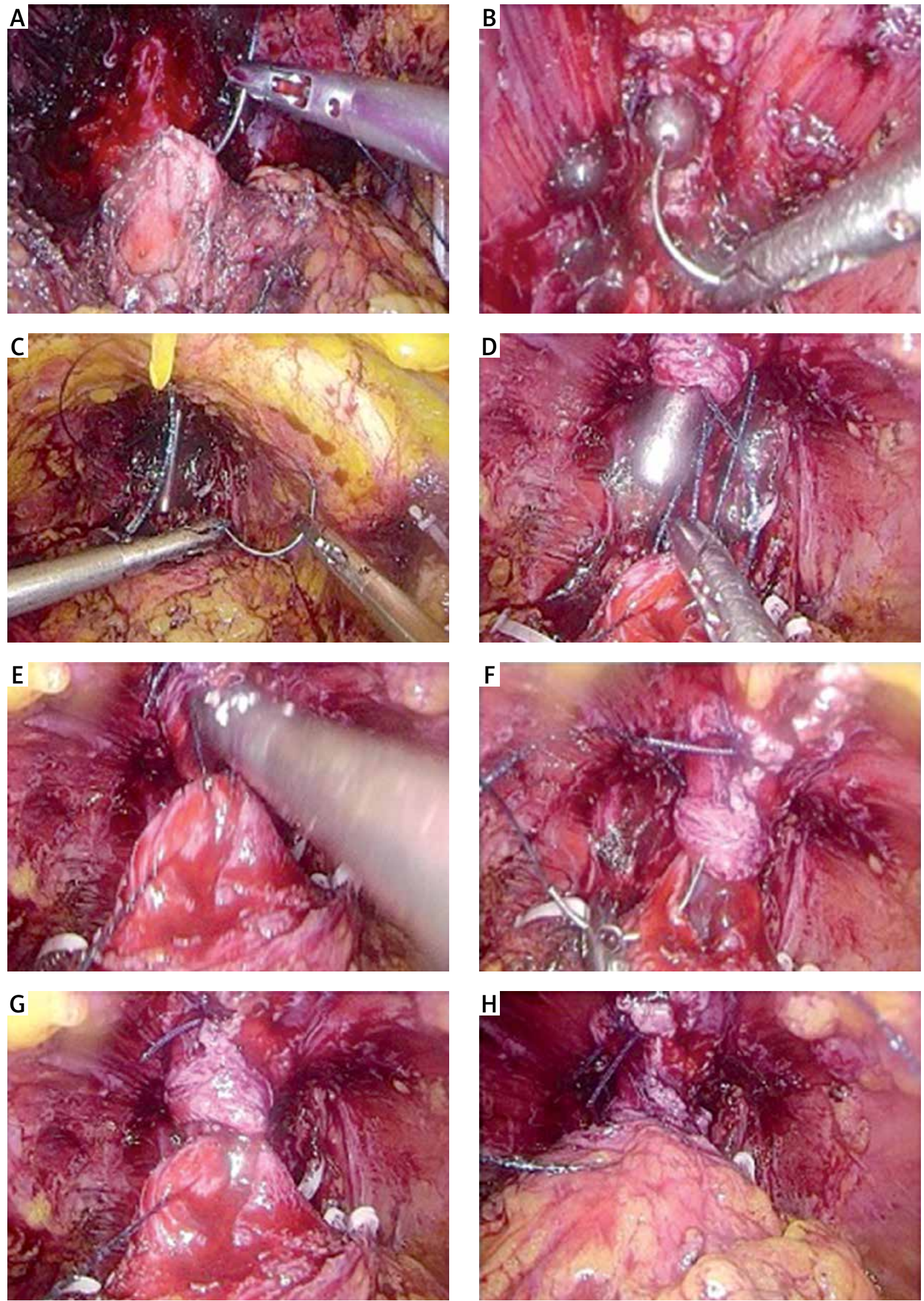

Photo 3. Vesicourethral anastomosis (VUA) technique with the V-Loc suture 
the Kruskal-Wallis rank sum test, $\chi^{2}$ test, or Fisher's exact test as appropriate. A two-sided $\alpha$ level of 0.05 was used as a cutoff for statistical significance. Continuous variables were analyzed without any arbitrary categorization.

The association between the time of anastomosis and the suturing technique was investigated using a multiple linear regression model, with the type of suture and log-transformed size of the prostate in grams as predictors and the anastomosis time as the dependent variable. Potential violations of the model's assumptions were inspected using diagnostic plots of the distribution of residuals.

A doubly robust estimation procedure was used with a simultaneous propensity score weighting and covariate adjustment in a logistic regression model to estimate the average treatment effect of each suture type on the occurrence of complications up to 90 days after surgery. Under the assumption of exchangeability, this method answered the question about the average relative effects of all three suturing techniques in the population after we controlled for pretreatment imbalances on observed variables. Generalized boosted models with 30000 trees were used for estimating propensity scores with the following variables: age, body mass index (BMI), clinical stage of the disease, Gleason grade (5 Grade Group system), log-transformed value of the prostate-specific antigen measurement, and size of the prostate in grams. Balance across treatment groups was checked visually with standardized effect size plots. Lingering imbalances between the groups were accounted for by using direct covariate adjustment in the final multiple logistic regression model.

The difference in time to recovery of urinary continence up to 1 year after surgery between the groups was studied using restricted mean survival time with covariate adjustment to control for potential confounding. We compared the mean time to recovery of urinary continence between Chlosta's running suture, the Van Velthoven suture, and the V-Loc after adjusting for age, BMI, and size of the prostate. All analyses were performed with $\mathrm{R}$ version 3.6.2.

\section{Results}

A total of 504 consecutive patients who underwent LRP for PCa between February 2014 and October 2018 in a single tertiary care center were enrolled in the study. The median age was 63 years
(IQR: 58-68), the median BMI was 27 (IQR: 25-30), and the average UCSF-Capra (University of California, San Francisco, Cancer of the Prostate Risk Assessment) score was 3 (IQR: 2-5). Of these patients, 109 received Chlosta's running suture, 117 the Van Velthoven suture, and 278 the V-Loc suture. Characteristics of the groups are presented in Table I. There were differences between groups regarding age, comorbidities (i.e., Charlson and American Society of Anesthesiologists score), clinical stage of the disease, Biopsy International Society of Urological Pathology (ISUP) score, and prostate volumes. Perioperatively, the groups were significantly different concerning time to catheter removal, intraoperative leaks, and percentage of clinically significant anastomotic leakage. During follow-up, differences were observed between groups for percentages of patients who developed BNC (Table II).

The median time of anastomosis and the median time of surgery were 13 (IQR: 10-16) $\mathrm{min}$ and 150 (IQR: 120-220) min, respectively, in the Chlosta's running suture group; 28 (IQR: 24-30) $\mathrm{min}$ and 175 (IQR: 150-205) min, respectively, in the Van-Velthoven suture group; and 12 (IQR: 11-16) min and 140 (IQR: 115-180) min, respectively, in the V-Loc suture group $(p<0.001)$ (Figure 2, Table II).

After adjustment for the size of the prostate after surgery in a linear regression model with a log-transformed dependent variable, the estimated time of anastomosis with the Van Velthoven suture was $105.6 \%$ longer (95\% confidence interval (Cl): 88.1124.7) than that required for completing the procedure using either Chlosta's running suture or the V-Loc. We did not find sufficient evidence to claim that the time of anastomosis was different when using V-Loc than it was when using Chlosta's running suture, with a point estimate indicating a 1.7\% prolongation of the procedure with V-Loc $(95 \% \mathrm{Cl}$ : $-5.7 \%$ to $9.7 \%$ ), and we found no evidence for operator bias in interaction tests (all $p s>0.05$ ).

Complications up to 90 days after surgery occurred in 20 (18.3\%) patients in the Chlosta's running suture group, in $27(23.1 \%)$ patients in the Van Velthoven group, and in $37(13.3 \%)$ patients in the V-Loc group ( $p=0.051)$ (Table II).

Generalized boosted models were used to estimate the propensity score weights for receiving one of the three sutures for every patient. After confirming convergence of each of the three model fits, we visually checked the balance between groups (Photo 1 , 
Table I. Baseline patient characteristics

\begin{tabular}{|c|c|c|c|c|}
\hline Parameter & $\begin{array}{l}\text { Chlosta's running } \\
\text { suture }\end{array}$ & $\begin{array}{l}\text { Van Velthoven } \\
\text { suture }\end{array}$ & V-Loc & $P$-value \\
\hline N & 109 & 117 & 278 & \\
\hline Age (median [IQR]) & $63[58-66]$ & 62. [58-66] & $64[59-68.75]$ & 0.039 \\
\hline Preop PSA (median [IQR]) & $8.65[6.28-12.25]$ & $7.74[6.0-10.2]$ & $8.81[6.5-13.23]$ & 0.088 \\
\hline BMI (median [IQR]) & $27.1[25.3-30]$ & $27.44[24.90-29.41]$ & $27[25-30]$ & 0.992 \\
\hline Charlson Comorbidity Index (median [IQR]) & $2.0[2.0-2.0]$ & $2.0[2.0-2.0]$ & $3.0[2.0-4.0]$ & $<0.001$ \\
\hline UCSF-Capra score (median [IQR]) & $3.0[2.00-4.00]$ & $3.0[2.0-4.0]$ & $3.0[2.0-5.0]$ & 0.12 \\
\hline ASA score, $n(\%)$ : & & & & 0.048 \\
\hline 1 & $2(1.8)$ & $7(6.0)$ & $14(5.0)$ & \\
\hline 2 & $100(91.7)$ & $100(85.5)$ & $223(80.2)$ & \\
\hline 3 & $7(6.4)$ & $10(8.5)$ & $41(14.7)$ & \\
\hline Prostate volume (TRUS) (median [IQR]) & $34.0[28.0-43.0]$ & $35.0[27.0-47.0]$ & $40.0[33.0-50.0]$ & $<0.001$ \\
\hline Clinical stage, $n(\%)$ : & & & & 0.027 \\
\hline cT1b & $0(0.0)$ & $0(0.0)$ & $1(0.4)$ & \\
\hline CT1c & $70(64.2)$ & $68(58.1)$ & $159(57.2)$ & \\
\hline cT2a & $15(13.8)$ & $5(4.3)$ & $8(2.9)$ & \\
\hline cT2b & $15(13.8)$ & $26(22.2)$ & $34(12.2)$ & \\
\hline CT2C & $8(7.3)$ & $13(11.1)$ & $34(12.2)$ & \\
\hline CT3 & $1(0.9)$ & $5(4.3)$ & $39(14.0)$ & \\
\hline CT4 & $0(0.0)$ & $0(0.0)$ & $3(1.1)$ & \\
\hline Biopsy ISUP grade group, $n$ (\%): & & & & $<0.001$ \\
\hline ISUP 1 & $68(62.4)$ & $78(66.7)$ & $112(40.3)$ & \\
\hline ISUP 2 & $20(18.3)$ & $25(21.4)$ & $89(32.0)$ & \\
\hline ISUP 3 & $12(11.0)$ & $6(5.1)$ & $33(11.9)$ & \\
\hline ISUP 4 & $9(8.3)$ & $7(6.0)$ & $31(11.2)$ & \\
\hline ISUP 5 & $0(0.0)$ & $1(0.9)$ & $13(4.7)$ & \\
\hline
\end{tabular}

IQR - interquartile range, preop. PSA - preoperative prostate-specific antigen, BMI - body mass index, ASA - American Society of Anesthesiology, UCSF - Capra score - University of California, San Francisco, Cancer of the Prostate Risk Assessment, TRUS - transrectal ultrasound, ISUP - International Society of Urological Pathology.

Figure 1). The average treatment effect of the suturing technique on the occurrence of complications, up to 90 days after the procedure, was estimated with a multiple logistic regression model weighted by the propensity score by using doubly robust estimation. The model summary is presented in Table III in the form of coefficients and corresponding standard errors. Estimated effects of interest, namely, the odds ratios for complications, were 1.65 (95\% $\mathrm{Cl}$ : 0.76-3.61) for the Van Velthoven suture and 0.90 (95\% Cl: 0.44-1.81) for the V-Loc suture compared with Chlosta's running suture.
In the final pathology results, there were differences regarding the final ISUP score and lymph node metastases (Table IV).

Unadjusted comparisons of the percentage of patients achieving urinary continence between groups are shown in Table V. Time to recovery of urinary continence after catheter removal (Figure 3), stratified by suture type, is shown in Figure 4. Data on urinary continence were missing in 24 patients who received the $\mathrm{V}$-Loc suture. After we adjusted for age, BMI, and prostate size, the time to urinary continence recovery was on average 19 days short- 
Table II. Intraoperative, perioperative, and postoperative characteristics

\begin{tabular}{|c|c|c|c|c|}
\hline Parameter & $\begin{array}{l}\text { Chlosta's running } \\
\text { suture }\end{array}$ & $\begin{array}{l}\text { Van Velthoven } \\
\text { suture }\end{array}$ & V-Loc & $P$-value \\
\hline N & 109 & 117 & 278 & \\
\hline Prostate volume [g] (median [IQR]) & $40[28.2-47]$ & $42.32[31.8-56]$ & $41[34-51]$ & 0.042 \\
\hline Anastomosis time [min] (median [IQR]) & $13[10-16]$ & $28[24-30]$ & 12 [11-16] & $<0.001$ \\
\hline Surgery time (median [IQR]) & $150[120-220]$ & 175 [150-205] & $140[115-180]$ & $<0.001$ \\
\hline Clinically significant anastomotic leak = $1(\%)$ & $1(0.9)$ & $3(2.6)$ & $5(1.8)$ & 0.740 \\
\hline Bladder neck contracture = $1(\%)$ & $4(3.7)$ & $16(13.7)$ & $12(4.3)$ & 0.001 \\
\hline Intraoperative anastomotic leak = 1 (\%) & $9(8.3)$ & $11(9.4)$ & $7(2.5)$ & 0.007 \\
\hline Time to catheter removal [days] (median [IQR]) & 15 [14-17] & $16(15-19)$ & $13(11-15)$ & $<0.001$ \\
\hline $\begin{array}{l}\text { Extraperitoneal access }=1 \\
\text { Transperitoneal access }=2=1 / 2(\%)\end{array}$ & $78 / 31(71.6 / 28.4)$ & $107 / 9(92.2 / 7.8)$ & $175 / 103(62.9 / 37.1)$ & $<0.001$ \\
\hline Intraoperative complications = $1(\%)$ & $6(5.6)$ & $2(1.7)$ & $7(2.5)$ & 0.220 \\
\hline Complications up to 90 days $=1(\%)$ & 20 (18.3) & $27(23.1)$ & 37 (13.3) & 0.051 \\
\hline
\end{tabular}

$I Q R$ - interquartile range.

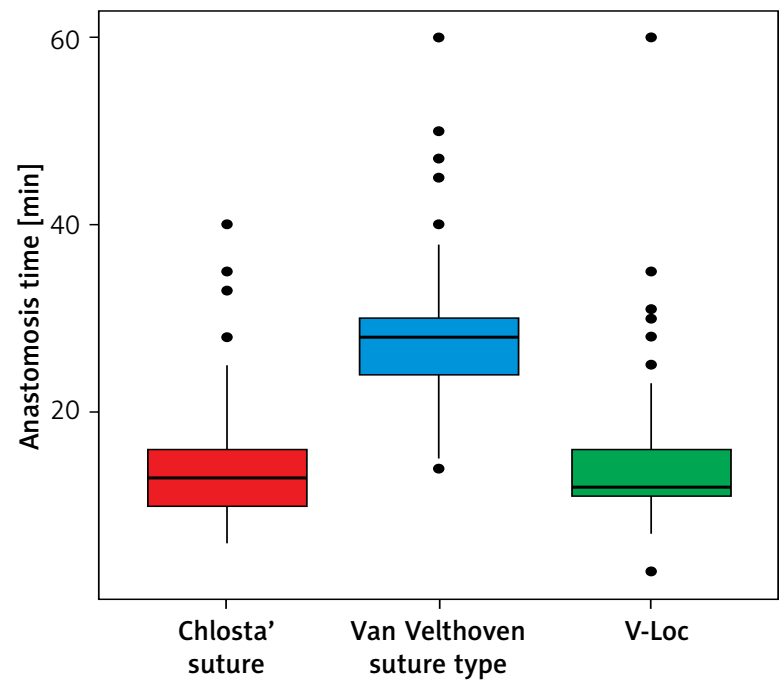

Figure 2. Time of anastomosis with different suturing techniques. Tukey's boxplots with vertical black lines indicating median values

er (95\% Cl: 5-33) when the V-Loc suture was used and 31 days shorter $(95 \% \mathrm{Cl}: 16-45)$ during 1 year of observation when the V-Loc suture was used than when the Van-Velthoven suture or Chlosta's running suture was used.

\section{Discussion}

This study did not show significant differences in the percentages of continent patients between three different VUA type groups at 12 and 18 months after surgery. The results were comparable with previously published data [20], which suggests that regardless of the applied VUA technique, the patients' long-term functional outcomes for urinary continence were satisfactory. However, the V-Loc group had faster continence recovery. Similar results were obtained in a small prospective study in patients with PCa who underwent robot-assisted radical prostatectomy [21]. Patients in the barbed suture VUA group had faster continence recovery in comparison with those in the classic Van Velthoven VUA group [21]. Interestingly, the difference in percentages of continent patients between VUA groups was also transient. According to that systematic review [21], the incidence of urinary leakage after LRP ranged from $3.2 \%$ to $33 \%$. In the current study, the percentage of clinically significant urinary leakage was comparable in all VUA groups and did not exceed $2 \%$ for the entire cohort. However, definitions of urinary leakage differ between studies [22, 23] and the percentage of urinary leakage in the current study might be underestimated, as it was limited to patients with a drainage output of over $100 \mathrm{ml}$ and an elevated creatinine level in the drained fluid.

In the Van Velthoven group, anastomosis time was longer than in the other two VUA groups, as was operating time. This might be a result of the more challenging technique of the Van Velthoven 
VUA and the requirement for simultaneous control of two threads. We found no significant differences between groups concerning complications up to 90 days after LRP, which is consistent with previous findings [24].

Data concerning the incidence of BNC after LRP are limited, but published reports show comparable results to those of our study [25-30]. In the Van Velthoven group, the incidence of BNC was high. This is a novel finding and no published data show similar results. A possible explanation is the increased tension in the Van Velthoven VUA or the greater number of needle passes, both of which might be linked with BNC [31-35].

In this study, the majority of patients underwent V-Loc VUA and the patients in that group had relatively unfavorable clinical risk factors and preoperative disease characteristics. The VUA type was chosen in accordance with the surgeon's preferences, which might suggest that in more complicated cases, surgeons felt more comfortable choosing V-Loc VUA. The decision on time to catheter removal was also made by the surgeon. Catheterization time was shorter in the V-Loc group, which might suggest that surgeons felt more confident about the V-Loc VUA.

This study had several limitations. First, the estimated effects of suture types on the occurrence of complications could be viewed as causal only if the assumption of exchangeability (i.e., no unobserved confounding) was held; this warrants further inves-

Table III. Doubly robust estimates from a weighted multiple logistic regression model

\begin{tabular}{|lcc|}
\hline Variable & Estimate & Standard error \\
\hline Van Velthoven suture & 0.50238 & 0.39811 \\
\hline V-Loc suture & -0.10980 & 0.35882 \\
\hline Age & 0.05909 & 0.02322 \\
\hline $\begin{array}{l}\text { Charlson Comorbidity } \\
\text { Index }\end{array}$ & -0.11355 & 0.14557 \\
\hline BMI & 0.02674 & 0.03519 \\
\hline Clinical stage T2 & 0.12024 & 0.31718 \\
\hline Clinical stage T3/T4 & -0.31503 & 0.49405 \\
\hline Log of PSA [ng/ml] & 0.05808 & 0.24822 \\
\hline ISUP 2 & 0.36371 & 0.34102 \\
\hline ISUP 3 & 0.44920 & 0.49149 \\
\hline ISUP 4 & -0.24778 & 0.56767 \\
\hline ISUP 5 & 0.50253 & 0.89347 \\
\hline Log of prostate size [g] & 0.11491 & 0.32657 \\
\hline Intercept & -6.66800 & 2.00681 \\
\hline
\end{tabular}

BMI-body mass index, PSA - prostate-specific antigen, ISUP-International Society of Urological Pathology.

Table IV. Pathological characteristics

\begin{tabular}{|c|c|c|c|c|}
\hline Parameter & $\begin{array}{c}\text { Chlosta's running } \\
\text { suture }\end{array}$ & $\begin{array}{l}\text { Van Velthoven } \\
\text { suture }\end{array}$ & V-Loc & $P$-value \\
\hline Pathological T-stage, $n(\%)$ : & & & & 0.279 \\
\hline pT2 & $45(41.3)$ & $50(42.7)$ & $108(38.8)$ & \\
\hline рT3a & $54(49.5)$ & $52(44.4)$ & $117(42.1)$ & \\
\hline pT3b & $9(8.3)$ & $15(12.8)$ & $48(17.3)$ & \\
\hline pT4 & $1(0.9)$ & $0(0.0)$ & $5(1.8)$ & \\
\hline Pathological N-stage 0/1 = $1(\%)$ & $1(0.9)$ & $0(0.0)$ & $33(11.9)$ & $<0.001$ \\
\hline Final ISUP grade group, $n$ (\%): & & & & 0.005 \\
\hline ISUP 1 & $45(41.3)$ & $38(32.5)$ & $66(23.9)$ & \\
\hline ISUP 2 & $40(36.7)$ & $45(38.5)$ & $124(44.9)$ & \\
\hline ISUP 3 & $15(13.8)$ & $26(22.2)$ & $45(16.3)$ & \\
\hline ISUP 4 & $3(2.8)$ & $6(5.1)$ & $15(5.4)$ & \\
\hline ISUP 5 & $6(5.5)$ & $2(1.7)$ & $26(9.4)$ & \\
\hline Extracapsular extension, $n(\%)$ & $64(58.7)$ & $66(56.4)$ & $168(60.6)$ & 0.730 \\
\hline Positive surgical margin, $n(\%)$ & $44(40.4)$ & $43(36.8)$ & $103(37.1)$ & 0.809 \\
\hline Positive apical margin, $n(\%)$ & 25 (22.9) & 25 (21.4) & 58 (20.9) & 0.905 \\
\hline
\end{tabular}

ISUP - International Society of Urological Pathology. 
Table V. Urinary continence outcomes

\begin{tabular}{|lcccc|}
\hline Return of continence [month] & $\begin{array}{c}\text { Chlosta's running } \\
\text { suture }\end{array}$ & $\begin{array}{c}\text { Van Velthoven } \\
\text { suture }\end{array}$ & V-Loc & $P$-value \\
\hline 3 months urinary continence, $n(\%)$ & $62(56.9)$ & $83(70.9)$ & $210(82.7)$ & $<0.001$ \\
\hline 6 months urinary continence, $n(\%)$ & $90(82.6)$ & $100(85.5)$ & $237(93.7)$ & 0.003 \\
\hline 12 months urinary continence, $n(\%)$ & $97(89.0)$ & $106(90.6)$ & $238(94.1)$ & 0.207 \\
\hline 18 months urinary continence, $n(\%)$ & $97(89.0)$ & $107(91.5)$ & $239(94.5)$ & 0.172 \\
\hline
\end{tabular}

Ordinal variables with more than three categories were compared using the Kruskal-Wallis test.

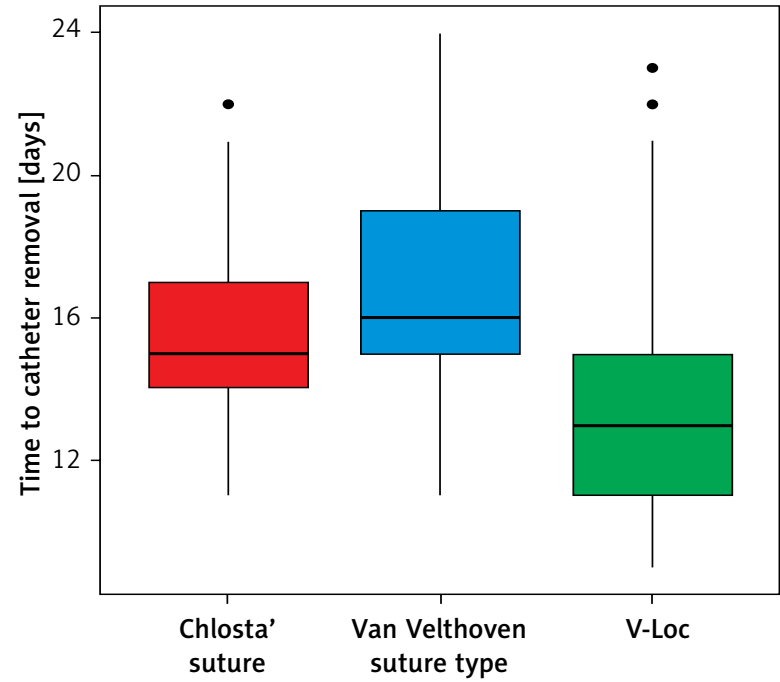

Figure 3. Time to catheter removal after different suturing techniques

tigation in an experimental setting. Second, with the sample size of this study, we were not able to rule out clinically relevant harm or benefit from using the studied suturing techniques because of the wide confidence intervals of the estimated treatment effects. Third, more sophisticated statistical modeling techniques such as restricted cubic splines or polynomials and the use of bootstrapping could have improved the quality of our inference even further. Fourth, this was a single-center study, which may limit the generalizability of our results.

The strength of this study was the consecutive sampling, which should provide adequate representativeness of the target population. We used doubly robust estimation, which protected against potential misspecification of either the propensity score model or the multiple logistic regression model. Finally, this was a pragmatic study that aimed to answer a clinically important question about the relative effects of three commonly used suturing techniques.

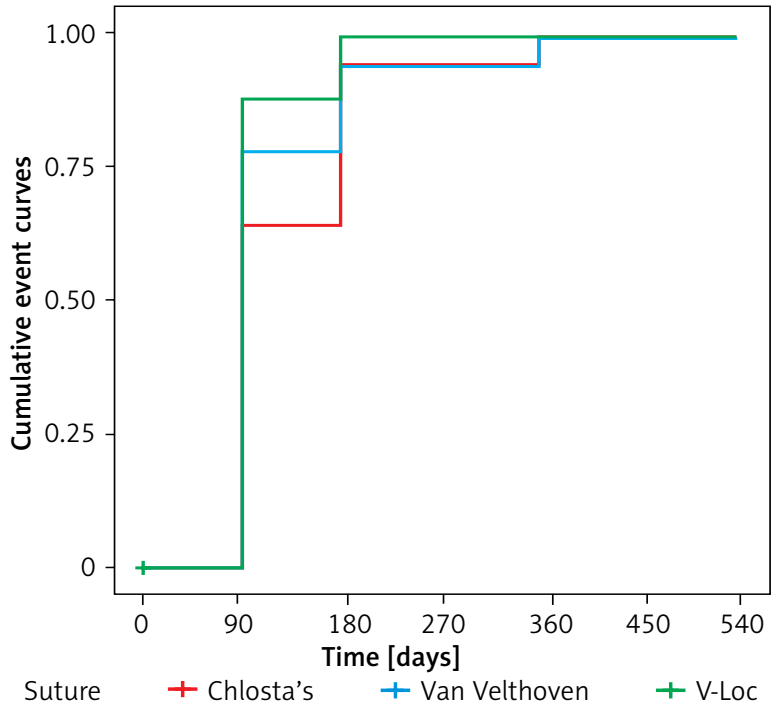

Figure 4. Cumulative event curves - recovery of urine continence

\section{Conclusions}

The current study showed comparable results concerning urinary continence at 12 and 18 months after LRP in all VUA groups. Data indicated faster continence recovery in the V-Loc group. The Van Velthoven VUA was more time-consuming and might be linked with an increased incidence of BNC.

\section{Conflict of interest}

The authors declare no conflict of interest.

\section{References}

1. Boyle P, Ferlay J. Cancer incidence and mortality in Europe, 2004. Ann Oncol 2005; 16: 481-8.

2. Haas GP, Delongchamps N, Brawley OW, et al. The worldwide epidemiology of prostate cancer: perspectives from autopsy studies. Can J Urol 2008; 15: 3866-71.

3. Schuessler WW, Schulam PG, Clayman RV, Kavoussi LR. Laparoscopic radical prostatectomy: initial short-term experience. Urology 1997; 50: 854-7. 
4. Rozet F, Fournier G, Cathelineau X, et al. Vesico-urethral anastomosis during total laparoscopic prostatectomy. Ann Urol 2006; 40: 50-6.

5. Stolzenburg J, Do M, Pfeiffler H, et al. The endoscopic extraperitoneal radical prostatectomy (EERPE): technique and initial experience. World J Urol 2002; 20: 48-55.

6. Jarzemski P, Listopadzki S, Słupski P, et al. Laparoscopic radica prostatectomy and extended pelvic lymph node dissection: a combined technique. Videosurgery Miniinv 2020; 15: 192-8.

7. Katz R, Nadu A, Olsson LE, et al. A simplified 5-step model for training laparoscopic urethrovesical anastomosis. J Urol 2003; 169: 2041-4.

8. Van Velthoven RF, Ahlering TE, Peltier A, et al. Technique for laparoscopic running urethrovesical anastomosis: the single knot method. Urology 2003; 61: 699-702.

9. Branco AW, Kondo W, de Camargo AH, et al. Laparoscopic running urethrovesical anastomosis with posterior fixation. Urology 2007; 70: 799-802.

10. Zarrelli G, Mastroprimiano G, Giovannone R, et al. Knotless "threeU-stitches" technique for urethrovesical anastomosis during laparoscopic radical prostatectomy. Int J Urol 2013; 20: 441-4.

11. Chłosta PL, Drewa T, Jaskulski J, et al. Bladder neck preservation during classic laparoscopic radical prostatectomy - point of technique and preliminary results. Videosurgery Miniinv 2012 7: 89-95.

12. Hoznek A, Salomon L, Rabii R, et al. Vesicourethral anastomosis during laparoscopic radical prostatectomy: the running suture method. J Endourol 2000; 14: 749-53.

13. Golabek T, Jarecki P, Jaskulski J, et al. Modified technique for laparoscopic running vesicourethral anastomosis. Videosurgery Miniinv 2014; 9: 357-61.

14. Poulakis V, Skriapas K, de Vries R, et al. Vesicourethral anastomosis during endoscopic extraperitoneal radical prostatectomy: a prospective comparison between the single-knot running and interrupted technique. Urology 2006; 68: 1284-9.

15. Simone G, Papalia R, Ferriero M, et al. Laparoscopic "single knot-single running" suture vesico-urethral anastomosis with posterior musculofascial reconstruction. World J Urol 2012; 30: 651-7.

16. Yang J, Shao PF, LV Q, et al. Continuous suture of a single absorbable suture: a new simplified vesicourethral anastomosis technique in laparoscopic radical prostatectomy. Int Surg 2014; 99: 656-61.

17. Golabek T, Jarecki P, Jaskulski J, et al. Modified technique for laparoscopic running vesicourethral anastomosis. Videosurgery Miniinv 2014; 9: 357-61.

18. Wiatr T, Golabek T, Dudek P, et al. Single running suture versus single-knot running suture for vesicourethral anastomosis in laparoscopic radical prostatectomy: a prospective randomised comparative study. Urol Int 2015; 95: 445-51.

19. Dindo D, Demartines N, Clavien PA. Classification of surgical complications: a new proposal with evaluation in a cohort of 6336 patients and results of a survey. Ann Surg 2004; 240: 205-13.

20. Ficarra V, Sooriakumaran P, Novara G, et al. Retropubic, laparoscopic, and robot-assisted radical prostatectomy: a systematic review and cumulative analysis of comparative studies. Eur Urol 2009; 55: 1037-63.
21. Manganiello M, Kenney P, Canes D, et al. Unidirectional barbed suture versus standard monofilament for urethrovesical anastomosis during robotic assisted laparoscopic radical prostatectomy. Int Braz J Urol 2012; 38: 89-96.

22. Mochtar CA, Kauer PC, Laguna MP, de la Rosette JJ. Urinary leakage after laparoscopic radical prostatectomy: a systematic review. J Endourol 2007; 21: 1371-9.

23. Hora M, Stránský P, Klečka J, et al. Managing urine leakage following laparoscopic radical prostatectomy with active suction of the prevesical space. Videosurgery Miniinv 2013; 8: 49-54.

24. Liao X, Qiao P, Tan Z, et al. "Total reconstruction" of the urethrovesical anastomosis contributes to early urinary continence in laparoscopic radical prostatectomy. Int Braz J Urol 2016; 42: 215-22.

25. Williams SB, Alemozaffar M, Lei Y, et al. Randomized controlled trial of barbed polyglyconate versus polyglactin suture for robot-assisted laparoscopic prostatectomy anastomosis: technique and outcomes. Eur Urol 2010; 58: 875-81.

26. Breyer BN, Davis CB, Cowan JE, et al. Incidence of bladder neck contracture after robot-assisted laparoscopic and open radical prostatectomy. BJU Int 2010; 106: 1734-8.

27. Besarani D, Amoroso P, Kirby R. Bladder neck contracture after radical retropubic prostatectomy. BJU Int 2004; 94: 1245-7.

28. Park R, Martin S, Goldberg JD, Lepor H. Anastomotic strictures following radical prostatectomy: insights into incidence, effectiveness of intervention, effect on continence, and factors predisposing to occurrence. Urology 2001; 57: 742-6.

29. Sujenthiran A, Cathcart P, van der Meulen J, et al. Quantifying severe urinary complications after radical prostatectomy: the development and validation of a surgical performance indicator using hospital administrative data. BJU Int 2017; 120: 219-25.

30. Tan G, Srivastava A, Grover S, et al. Optimizing vesicourethral anastomosis healing after robot-assisted laparoscopic radical prostatectomy: lessons learned from three techniques in 1900 patients. J Endourol 2010; 24: 1975-83.

31. Borboroglu PG, Sands JP, Roberts JL, Amling CL. Risk factors for vesicourethral anastomotic stricture after radical prostatectomy. Urology 2000; 56: 96-100.

32. Cho HJ, Jung TY, Kim DY, et al. Prevalence and risk factors of bladder neck contracture after radical prostatectomy. Korean J Urol 2013; 54: 297-302.

33. Msezane LP, Reynolds WS, Gofrit ON, et al. Bladder neck contracture after robot-assisted laparoscopic radical prostatectomy: evaluation of incidence and risk factors and impact on urinary function. J Endourol 2008; 22: 97-104.

34. Moran ME, Marsh C, Perrotti M. Bidirectional-barbed sutured knotless running anastomosis $v$ classic Van Velthoven suturing in a model system. J Endourol 2007; 21: 1175-8.

35. Lee SW, Han DH, Lee KS, Jeon SS. Effect of continuous urethro-vesical anastomosis technique in incontinence after radical retropubic prostatectomy, 1:1 matching study. Int Neurourol J 2015; 19: 113-9.

Received: 4.01.2021, accepted: 21.03.2021. 